\title{
The Hitchhickers Guide to the Integers: Die Sache mit der 42
}

\author{
Konrad Krug
}

Die Zahlentheorie nimmt in der Mathematik in einiger Hinsicht eine Sonderstellung ein: Obwohl sie sich, grob gesagt, mit den ganzen Zahlen beschäftigt, also dem Anschein nach leicht zu verstehenden Objekten, gilt sie bei vielen als kompliziertestes Teilgebiet der Mathematik. Dies liegt nicht zuletzt daran, dass bei der Untersuchung zahlentheoretischer Probleme Methoden aus nahezu allen Teilgebieten der Mathematik angewandt werden: So muss man, um in der Disziplin zu bestehen, Expertise in Geometrie, Algebra, Analysis, Topologie und vielen anderen mitbringen.

Ein weiteres Indiz für den scheinbaren Widerspruch zwischen der vordergründigen Einfachheit ihres Untersuchungsgegenstandes und dem tatsächlichen Schwierigkeitsgrad der Disziplin ist der Umstand, dass offene Probleme in der Zahlentheorie oftmals auch Laien zugänglich sind: Ist jede gerade Zahl Summe zweier Primzahlen (z. B. $100=47+53)$ ? Gibt es unendlich viele Primzahlzwillinge, das sind Primzahlpaare, deren Differenz zwei beträgt, wie z. B. 17 und 19? Gibt es natürliche Zahlen $x, y, z$ und $n$, sodass $x^{n}+y^{n}=z^{n}$ gilt?

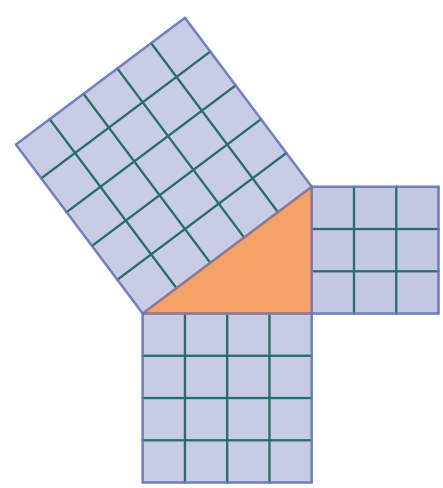

Für die Zahlen 3, 4, und 5 gilt: $3^{2}+4^{2}=5^{2}$. Aber gibt es solche Zahlentripel auch dann, wenn der Exponent größer ist als 2? Nein, behauptete Pierre de Fermat im 17. Jahrhundert. Bewiesen wurde diese Aussage erst 300 Jahre später.

Während es sich bei den ersten beiden Fragen nach wie vor um offene Probleme handelt, zeigte der Brite Andrew Wiles 1994 in einem über 90 Seiten langen Beweis, dass das letztgenannte Problem, abgesehen von trivialen Ausnahmen mit Einsen und Nullen für $n>2$ keine Lösungen hat. Die Lösung dieses vormals Fermatsche Vermutung genannten Problems war über Jahrhunderte hinweg eine der wichtigsten offenen Fragen der Mathematik, an der sich in der Geschichte nahezu jede/r große Mathematiker/in vergeblich versucht hatte. (Übrigens: In seiner bahnbrechenden Arbeit bewies Wiles die Fermatsche Vermutung nicht direkt, sondern zeigte vielmehr einen tiefliegenden
Zusammenhang zwischen Modulformen und gewissen elliptischen Kurven auf - Fermats letzter Satz ist eine der zahlreichen Folgerungen aus diesem Modularitätssatz genannten Resultat).

Gleichungen der Form $x^{n}+y^{n}=z^{n}$ mit Lösungen in den ganzen Zahlen sind Beispiele für sogenannte diophantische Gleichungen, das sind Polynomialgleichungen in einer oder mehreren Variablen, mit der Zusatzbedingung, dass es sich bei den Lösungen um ganze Zahlen handeln muss. Die Theorie der Polynomialgleichungen ohne diese Zusatzbedingung ist in der algebraischen Geometrie seit Jahrhunderten gut erforscht (was natürlich nicht heißt, dass es keine offenen Fragen mehr gibt); im Gegensatz dazu stellen diophantische Gleichungen die mathematische Forschung vor massive Probleme: Nicht nur, dass häufig kein brauchbares Lösungsverfahren bekannt ist, allein die Frage nach der Existenz von Lösungen ist im Allgemeinen nicht zu beantworten: 1970 zeigte der Russe Juri Wladimirowitsch Matijassewitsch, dass es nicht möglich ist, einen Computer (oder auch einen mit Zettel und Bleistift ausgestatteten, sehr fleißigen Menschen) darauf zu trimmen, zu einer gegebenen diophantischen Gleichung zu entscheiden, ob sie lösbar ist oder nicht (genauer: Die Menge der Lösungsmengen Diophantischer Gleichungen ist genau die Menge der rekursiv aufzählbaren Mengen, und damit unentscheidbar). Dabei ist hervorzuheben, dass diese Unmöglichkeit nicht etwa aus den begrenzten Rechenkapazitäten heutiger oder zukünftiger Rechner resultiert, sondern prinzipieller Natur ist, vergleichbar mit der Tatsache, dass das Erreichen von Überlichtgeschwindigkeit keine Frage der Benzinmenge ist.

Es ist also schwierig mit den diophantischen Gleichungen. Umso bemerkenswerter ist es, dass es nun dem britischen Mathematiker Andrew Brooker gelungen ist, einem weiteren Problem aus der Theorie der diophantischen Gleichungen ein kleines Stückchen näher zu kommen: Dem Problem der drei Dreierpotenzen (engl. sums of three cubes). Kurios: Inspiriert wurde er dabei durch ein Video des Youtubekanals Numberphile, der sich auf populärwissenschaftliche Art mit Mathematik beschäftigt.

Bei dem Problem geht es um die Frage, welche ganzen Zahlen sich als Summe von drei Dreierpotenzen schreiben lassen. Derlei Zahlen zu konstruieren ist offenkundig nicht schwierig: Man erhält sie, indem man drei Dreierpotenzen addiert, zum Beispiel ist

$$
(-2)^{3}+3^{3}+(-1) 3=-8+27+-1=18,
$$

18 ist also Element der oben beschriebenen Zahlenmenge. Schwierig hingegen ist es, zu entscheiden, wann eine gegebene Zahl sich derart darstellen lassen kann: gehört 
die Zahl 10 dazu? Die 23? Wie sieht es mit der Zahl 33 aus?

Mit ein wenig modularer Arithmetik ist es möglich, eine große Gruppe von Zahlen auszuschließen: Man kann zeigen, dass es für Zahlen $n$, die bei Division mit 9 den Rest 4 oder 5 ergeben, keine Darstellung der Form $n=x^{3}+y^{3}+z^{3}$ geben kann. Dies betrifft zum Beispiel die Zahlen 4, 5, 13, 14, 22, 23, und so weiter. Für alle anderen ganzen Zahlen wird vermutet, dass solche Darstellungen stets existieren; leicht $\mathrm{zu}$ finden sind sie allerdings selten: die „einfachste“ Lösung für die Zahl 30 besteht zum Beispiel aus zwei zehnstelligen Zahlen und einer neunstelligen. Mit massivem Rechnereinsatz ist es gelungen, für fast alle Zahlen zwischen 1 und 1000 eine Lösung zu finden; ausgenommen ist eine Liste von 14 Zahlen, die sich beharrlich einer Darstellung als Summe dreier Kuben erwehrten.

Diese Liste war Stand der Forschung als der Mathematik-Vlogger Brady Haran vom Youtubekanal Numberphile ein Video mit dem Zahlentheoretiker Tim Browning veröffentlichte, in dem letzterer das Problem erklärte. "Don't pause the video and try yourself!" hieß es im Video, als die kleinste Zahl der Liste, die Zahl 33, diskutiert wurde; „Haltet dieses Video nicht an, um es selber auszuprobieren!“. Eine ungewöhnliche Warnung, die der sonst so auf Interaktion mit seinem Publikum bedachte Haran ausrief; der Grund dafür lag scheinbar auf der Hand: Kein/e Zuschauer/in sei in der Lage, dieses Problem ad hoc anzugehen; nicht, wenn Generationen von Zahlentheoretiker/innen und Informatiker/innen daran scheiterten. Doch wie bei jeder anderen Warnung gab es auch im Fall der Zahl 33 unerschrockene, die sich allen Unkenrufen zum Trotz dem Problem widmeten. Einer von ihnen war Andrew Booker.

Als hätte er die Warnung geflissentlich überlesen, machte sich der britische Mathematiker von der University of Bristol daran, das Rätsel um die Zahl 33 zu lösen. Dabei formulierte er das Problem mit Hilfe einfacher Arithmetik derart um, dass der benötigte Rechenaufwand klein genug wurde, um es mit dem Supercomputer seiner Universität anzugehen. Und tatsächlich: Nach wenigen Tagen, und damit viel früher als erwartet, gab der Rechner folgendes Ergebnis aus:

$$
\begin{aligned}
33=8866128975287528^{3} & \\
+(-87784054 & 42862239)^{3} \\
& +(-2736111468807040)^{3}
\end{aligned}
$$

Damit wurde die Liste der Zahlen, für die keine Darstellung als Summe dreier Kubikzahlen bekannt ist, um eins reduziert. Dass man eines Tages für jede Zahl, die bei Di- vision mit 9 nicht den Rest 4 oder 5 ergibt, eine solche Darstellung kennt, ist durch das Resultat freilich noch nicht abzusehen. Optimistisch stimmt jedoch, dass Booker mit seiner Berechnungsmethode Möglichkeiten aufgezeigt hat, wie die Komplexität des Problems drastisch reduziert werden kann, um somit einer effektiveren computergestützten Suche in den höchsten Höhen des Zahlenreiches den Weg zu ebnen.

Ob er seine Lösungszahlen auswendig aufsagen könne, fragt Haran. „Nein, das kann ich nicht“ entgegnet Booker und lacht. „Ich habe noch nicht einmal bemerkt, dass eine von ihnen eine Primzahl ist."

Damit wurde die 42 zur kleinsten Zahl, deren Darstellung als Summe dreier Kubikzahlen nicht bekannt war, ein Umstand, der die Fans des Literarischen Werkes Douglas Adams‘ entzücken dürfte, gilt sie doch in The Hitchhiker's Guide to the Galaxy als die Antwort auf die Frage nach dem Leben, dem Universum und dem ganzen Rest. Ob eine Darstellung für die Zahl 42 in naher Zukunft gefunden werden kann, war bis dahin völlig unklar. Diese Kuriosität unterstrich der User [SIC] unter dem Numberphilevideo mit seinem Kommentar: "Are you kidding me? We are literally in a situation where 42 is the answer but we will probably need to build a new supercomputer to figure out what the question is?"

Nur wenige Monate später allerdings ist es Booker gelungen, in Kooperation mit dem MIT-Mathematiker Andrew Sutherland auch für die Zahl 42 eine Darstellung als Summe dreier Kuben zu finden. Unterstützt wurden sie dabei von einem MIT-eigenen Rechnernetzwerk (welches zwar leistungsfähig, aber freilich nicht, wie Deep Thought, das „Vorbild“ aus Douglas Adams' Werk, von planetarer Größe ist), das nach mehr als einer Million Rechenstunden die Antwort

$$
\begin{aligned}
& 42=(-80538738812075974)^{3} \\
&+80435758145817515^{3} \\
&+12602123297335631^{3}
\end{aligned}
$$

ausgab. Damit ist es für jede Zahl unter 100 (abgesehen von solchen, für die es bewiesenermaßen unmöglich ist) gelungen, sie als Summe von drei Kubikzahlen zu schreiben.

Dieses mathematische Rätsel ist nun endgültig gelöst; die Frage nach dem Leben, dem Universum und dem ganzen Rest bleibt hingegen, abgesehen davon, dass die Antwort sicherlich 42 ist, offen. Und ob es nun darum geht, die Galaxis per Anhalter zu durchqueren oder diophantische Gleichungen zu lösen: In jedem Falle ist es ratsam, stets ein Handtuch dabeizuhaben. 